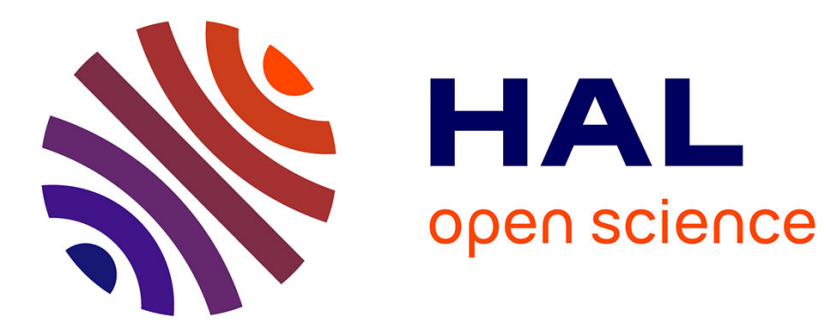

\title{
Topological optical isolator based on polariton graphene
} D. Solnyshkov, Olivier Bleu, Guillaume Malpuech

\section{To cite this version:}

D. Solnyshkov, Olivier Bleu, Guillaume Malpuech. Topological optical isolator based on polariton graphene. Applied Physics Letters, 2018, 112, pp.031106. 10.1063/1.5018902 . hal-01914233

\section{HAL Id: hal-01914233 \\ https://hal.uca.fr/hal-01914233}

Submitted on 19 Dec 2018

HAL is a multi-disciplinary open access archive for the deposit and dissemination of scientific research documents, whether they are published or not. The documents may come from teaching and research institutions in France or abroad, or from public or private research centers.
L'archive ouverte pluridisciplinaire HAL, est destinée au dépôt et à la diffusion de documents scientifiques de niveau recherche, publiés ou non, émanant des établissements d'enseignement et de recherche français ou étrangers, des laboratoires publics ou privés.

\section{다)(1) $\$$}

Distributed under a Creative Commons Attribution - NonCommercial| 4.0 International 


\section{Topological optical isolator based on polariton graphene}

D. D. Solnyshkov, O. Bleu, and G. Malpuech

Citation: Appl. Phys. Lett. 112, 031106 (2018); doi: 10.1063/1.5018902

View online: https://doi.org/10.1063/1.5018902

View Table of Contents: http://aip.scitation.org/toc/apl/112/3

Published by the American Institute of Physics

\section{Articles you may be interested in}

The interplay between excitons and trions in a monolayer of $\mathrm{MoSe}_{2}$

Applied Physics Letters 112, 031107 (2018); 10.1063/1.5019177

Polariton condensation in S- and P-flatbands in a two-dimensional Lieb lattice

Applied Physics Letters 111, 231102 (2017); 10.1063/1.4995385

Observation of valley-selective microwave transport in photonic crystals

Applied Physics Letters 111, 251107 (2017); 10.1063/1.5009597

High transmission in a metal-based photonic crystal

Applied Physics Letters 112, 013504 (2018); 10.1063/1.5006595

Broadband nonlinear optical response of monolayer $\mathrm{MoSe}_{2}$ under ultrafast excitation

Applied Physics Letters 112, 031108 (2018); 10.1063/1.5010060

Infrared emission of a freestanding plasmonic membrane

Applied Physics Letters 112, 031104 (2018); 10.1063/1.5017194

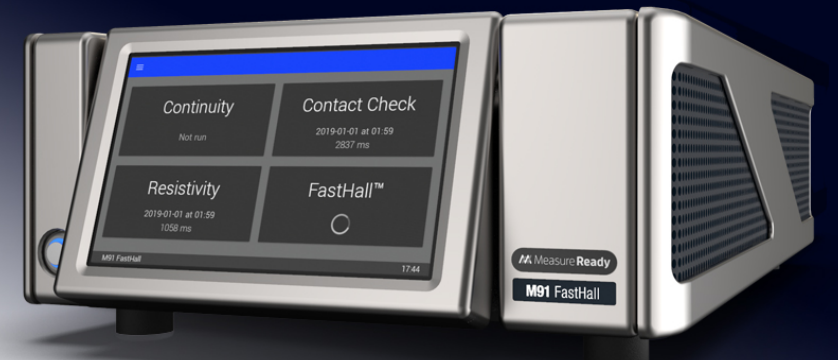

\section{now Measure Ready M91 FastHall ${ }^{\mathrm{TM}}$ Controller \\ A revolutionary new instrument for complete Hall analysis




\title{
Topological optical isolator based on polariton graphene
}

\author{
D. D. Solnyshkov, O. Bleu, and G. Malpuech \\ Institut Pascal, PHOTON-N2, University Clermont Auvergne, CNRS, 4 avenue Blaise Pascal, \\ 63178 Aubière Cedex, France
}

(Received 11 December 2017; accepted 4 January 2018; published online 17 January 2018)

\begin{abstract}
We propose a scheme of a topological optical isolator based on the quantum anomalous Hall effect with strongly coupled exciton-polaritons in a patterned GaAs cavity. We study the practical properties of such a device and optimize its parameters. We obtain an isolation ratio of $49 \mathrm{~dB}$ at a wavelength of $783 \mathrm{~nm}$ for a device of $40 \mu \mathrm{m}$ with a maximal signal modulation frequency of $300 \mathrm{GHz}$, operating at temperatures up to 50 K. Published by AIP Publishing. https://doi.org/10.1063/1.5018902
\end{abstract}

Topological insulators ${ }^{1}$ (TIs) represent a rapidly developing branch of modern Physics. The discovery of the Quantum Hall effect ${ }^{2}$ and the metallic surface states in certain insulating materials and their interpretation in terms of topological invariants ${ }^{3}$ have shown that the properties of periodic systems are determined not only by the dispersion and its filling but also by the eigenstates themselves, in particular, by their topology. In electronic systems, these effects mostly affect the conduction properties, which, in the case of the quantum spin-Hall effect, become spin-dependent. ${ }^{4}$

In optics, the motivation for the research on TIs or topological mirrors has mostly been the chirality of their edge states. ${ }^{5,6}$ Indeed, the reciprocal nature of conventional optics (Lorentz reciprocity) is a problem for optical logics and optical computation: any signal can be reflected at the receiver and affect the sender. Optical isolators ${ }^{7}$ based on the Faraday effect in magnetic fields are relatively difficult to integrate. Thus, the TIs with chiral edge states have been proposed as the solution of the reciprocity problem. The one-way nature of these states also provides a protection from backscattering, improving the transmission. Many devices based on the principles of topological photonics have been proposed and implemented recently: ${ }^{8-10}$ topological lasers, ${ }^{11,12}$ slow-lightbased topological devices,${ }^{13}$ one-way fibers, ${ }^{14}$ topologically protected delay lines, ${ }^{15,16}$ circulators, ${ }^{17}$ amplifiers, ${ }^{18}$ logical chips, ${ }^{19-21}$ topological switches, ${ }^{22,23}$ splitters, ${ }^{24-26}$ and, finally, optical isolators. ${ }^{27-29}$ In particular, a $50 \mathrm{~dB}$ isolation ratio has been predicted for a $7 \mathrm{~mm}$ size device, ${ }^{27}$ which is much smaller than the typical optical isolator, but still large for integrated optics.

Many works on chiral states in topological photonics based on different mechanisms have appeared in the last few years. ${ }^{30}$ The time-reversal symmetry may be broken either by a magnetic field or by other means,${ }^{31}$ like a nonlinear response $^{32,33}$ or a periodic space-time modulation. ${ }^{34}$ Often, photons are mixed with the excitations of matter (excitons, ${ }^{35}$ plasmons ${ }^{36}$ etc.) to enhance the symmetry-breaking mechanism. ${ }^{9}$ The periodic medium also varies: the examples include coupled waveguide arrays, ${ }^{37}$ photonic crystals, ${ }^{38}$ patterned cavities, ${ }^{39}$ and even Möbius microcavities. ${ }^{40}$ The new proposals for optical isolators have to be compared with the existing devices and with the proposals based on other mechanisms: mostly on the Faraday rotation effect, or, more generally, on time-reversal symmetry breaking by a magnetic field ${ }^{41}$ or by other means. ${ }^{42-44}$ Most of them are macroscopic structures, with few recent proposals oriented towards integrated photonics. ${ }^{45-48}$ The most recent works demonstrate microscopic structures for integrated silicon photonics. ${ }^{49}$ What distinguishes the topological structures is the complete chirality-impossibility of propagation in one of the two directions in space. Traditional isolators are rather based on destructive interference, for example, in coupled waveguides, ${ }^{50,51}$ and the proposals try to take advantage of novel media, such as graphene. ${ }^{47}$ The bandwidth of integrated topological optical isolators is much smaller than that of conventional ones, especially when the latter are specifically optimized. $^{52}$

However, so far, the practical questions of device geometry, signal injection, and outcoupling have not been sufficiently treated for topological optical isolators. Indeed, any practical application requires working with finite-size samples, where the edge states are present on all boundaries, and necessarily there are paths connecting any 2 points on the edge in both directions (like one can fly from Washington, DC, to Sydney via Los Angeles or from Sydney to Washington, DC, via Tokyo), which breaks down the oneway nature of the device as a whole. The practical applications also require efficient injection and extraction of the signal from the chiral states.

In this work, we propose a scheme of a topological optical isolator for integrated photonics based on a polariton graphene lattice with input and output channels. Our solution is based on the exciton-polariton platform ${ }^{53}$ (strong coupling of light and excitons) with periodic potential ${ }^{39,54-56}$ (created by patterning, acoustics, ${ }^{57,58}$ or optics ${ }^{59,60}$ ) which has been the subject of many recent proposals ${ }^{35,61-69}$ and experiments ${ }^{39,70-73}$ linked with topological photonics. In this system, the time-reversal symmetry breaking ${ }^{74}$ can be achieved either by an applied magnetic field ${ }^{35}$ (whose effect can be maximized by using diluted magnetic semiconductors) or by circular-polarized optical pumping. ${ }^{33}$ We analyze how the crucial properties of the optical isolator device depend on its geometry. This allows us to optimize the design of the device and obtain an isolation ratio of $49 \mathrm{~dB}$ and a transmission coefficient close to 1 .

The general scheme of the simplest device based on the edge states of a TI, a topological optical isolator, is shown in Fig. 1(a). Besides the TI itself (marked $\mathbb{Z}$ ), the device 

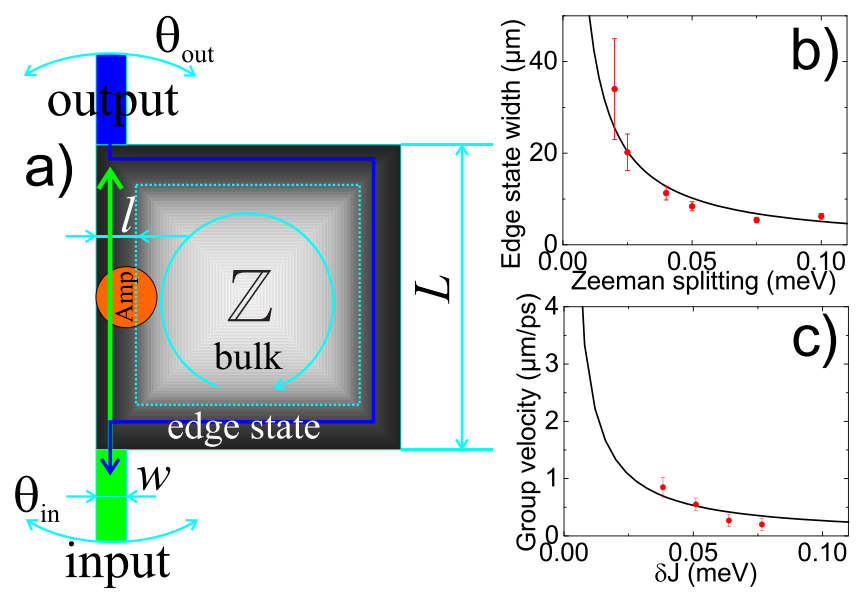

FIG. 1. (a) The scheme of the topological optical isolator with the main device parameters discussed in the text $\left(L, l, w, \theta_{\text {in }}\right.$, and $\left.\theta_{\text {out }}\right)$. (b) The width of the edge state as a function of Zeeman splitting $\Delta_{Z}$ for fixed TE-TM splitting $\delta J$. (c) The group velocity as a function of TE-TM splitting $\delta J$ for fixed Zeeman splitting $\Delta_{Z}$.

includes the input and output leads (waveguides). The main practical parameters are the device size $L$, the edge state spatial extension $l$, the input/output waveguide widths $w$, and their orientation angles $\theta_{\text {in/out }}$. The forward signal (green) and backward (reflected) signal (blue) follow different paths in real space because of the chiral nature of the edge states. As we shall show, the isolation ratio is induced by (1) the weak backward injection, (2) the longer backward path leading to higher radiative decay, and, optionally, (3) an external pump (orange spot marked "Amp"), which, due to the spatial separation of both paths, supports the forward transmission only, keeping it close to unity.

In our work, the TI is a honeycomb lattice of coupled pillar microcavities (radius of the pillars $r=1.5 \mu \mathrm{m}$, lattice parameter $a=2.5 \mu \mathrm{m}$, etched from a GaAs $\lambda / 2$ cavity with a Rabi splitting of $15 \mathrm{meV}$ ) in the strong exciton-photon coupling regime (the so-called polariton graphene ${ }^{39}$ ). We begin with a tight-binding description ${ }^{35}$ which allows obtaining the analytical estimations for the important geometrical parameters of the system. We include the light polarization degree of freedom and the so-called TE-TM spin-orbit coupling ${ }^{30}$ (present in any photonic system) in our model. The Hamiltonian is a $4 \times 4$ matrix written in the $\left(\Psi_{A}^{+}, \Psi_{A}^{-}, \Psi_{B}^{+}, \Psi_{B}^{-}\right)$basis

$$
H_{q a h}=\left(\begin{array}{ll}
\Delta_{z} \sigma_{z} & F_{k} \\
F_{k}^{\dagger} & \Delta_{z} \sigma_{z}
\end{array}\right), \quad F_{k}=-\left(\begin{array}{ll}
f_{k} J & f_{k}^{+} \delta J \\
f_{k}^{-} \delta J & f_{k} J
\end{array}\right),
$$

where $\sigma_{z}$ is the 3 rd Pauli matrix, $J \approx 0.25 \mathrm{meV}$ is the nearestneighbor tunneling coefficient, $\Delta_{z}$ is the Zeeman splitting, $\delta J$ is the spin orbit coupling strength, $f_{k}=\sum_{j=1}^{3} \exp \left(-i \mathbf{k} \mathbf{d}_{\phi_{j}}\right)$, and $f_{k}^{ \pm}=\sum_{j=1}^{3} \exp \left(-i\left[\mathbf{k} \mathbf{d}_{\phi_{j}} \mp 2 \phi_{j}\right]\right)\left(\mathbf{d}_{\phi_{j}}\right.$ is the vector linking the pillar $A$ with its neighbor $B_{j}$ and $\phi_{j}$ is the orientation of this vector).

When a gap is opened at the Dirac point, one can use the low-energy approximation and reduce the problem to the massive Dirac Hamiltonian at each avoided crossing (even in the case of trigonal warping ${ }^{75}$ ) where the mass $m$ is linked with the parameter determining the gap size. If $\Delta_{Z}<\delta J$, then the gap size is controlled by $\Delta_{Z}$, and the mass is ${ }^{75}$ $m=4 \hbar^{2} \Delta_{Z} / 9 a^{2} J^{2}$. This allows us to find one of the most important parameters, the characteristic spatial extension of the edge state $l$, crucial for the final device size. Since the low-energy Hamiltonian is parabolic in $\mathbf{q}=\mathbf{k}-\mathbf{K}$, the decay length is found as for the tunneling under a potential barrier of a height $\Delta_{Z}$ determining a characteristic wavevector $\kappa_{0}$

$$
\kappa_{0}=\frac{\sqrt{2 m \Delta_{Z}}}{\hbar}, \quad l=\frac{3 a J}{\Delta_{Z}} .
$$

We see that the Zeeman splitting needs to be increased if we want to reduce the size of the edge states and thus the device size. However, when the Zeeman splitting $\Delta_{Z}$ becomes comparable with $\delta J$, a topological transition takes place. For these values, our simplified expression does not hold any more. However, an estimate can still be obtained replacing $\Delta_{Z}$ by the gap size $E_{g}$ (from tight-binding calculations or experimental measurements). The results of the calculation of the edge state width $l$ as a function of the Zeeman splitting $\Delta_{Z}$ for fixed TE-TM splitting $\delta J$ are shown in Fig. 1(b). The analytical curve (black line) corresponds to Eq. (2).

Another parameter, which is clearly important for the functioning of the device, is the group velocity of the edge states, $v=\hbar^{-1} \partial E / \partial k$, determined by their dispersion. The latter can be estimated analytically from the low-energy analysis of the bulk dispersion in the absence and in the presence of the magnetic field. Indeed, as can be seen from Ref. 35 , the edge state covers the whole gap $\Delta_{Z}$ for the change of the wavevector corresponding to the characteristic scale of the trigonal warping, the latter being determined from the zero-field solution as $k_{0}=\delta \mathrm{J} / \mathrm{Ja},{ }^{76}$ and thus, the group velocity is $v \approx \Delta_{Z} J a / \hbar \delta J$. It is shown in Fig. 1(c) as a black line.

The overall optimization problem can be stated as follows. We would like to maximize the transmission in the forward direction (input $\rightarrow$ output, $T_{F}$ ) and minimize in the backward direction (output $\rightarrow$ input, $T_{B}$ ). Here, we would like to stress that for a finite size object, there is no real violation of reciprocity: the signals can travel both ways, and they are simply taking different paths with the lengths $l_{i \rightarrow o}$ and $l_{o \rightarrow i}$ on the opposite sides of the sample, which necessarily exist and are not located at infinity. Therefore, a signal can definitely get from the output to the input, but we would like it to be attenuated as much as possible due to the finite lifetime $\tau$ acting on the relative path difference. Neglecting the pump sustaining selectively the transmitted signal, the ratio of transmission coefficients, which corresponds to the ratio of intensities, reads

$$
\frac{T_{F}}{T_{B}}=\frac{I_{i \rightarrow o}}{I_{o \rightarrow i}}=\frac{K_{f} X_{f}}{K_{b} X_{b}} \exp \left(\frac{l_{o \rightarrow i}-l_{i \rightarrow o}}{v \tau}\right),
$$

where $v$ is the group velocity of the edge states, $K_{f}$ and $K_{b}$ are the forward and backward injection efficiency coefficients, and $X_{f}$ and $X_{b}$ are the forward and backward extraction efficiency coefficients.

The injection and extraction depend strongly on the ratio of $l$ and $w$. Let us begin with the simple configuration with straight leads $\left(\theta_{i n, \text { out }}=0\right)$. In the forward direction, based on the spatial distribution of intensities, the injection can be approximated by $K_{f}=\min (1, l / w)$ : it becomes reduced only if $w>l$. At the output, the conversion is performed in the 


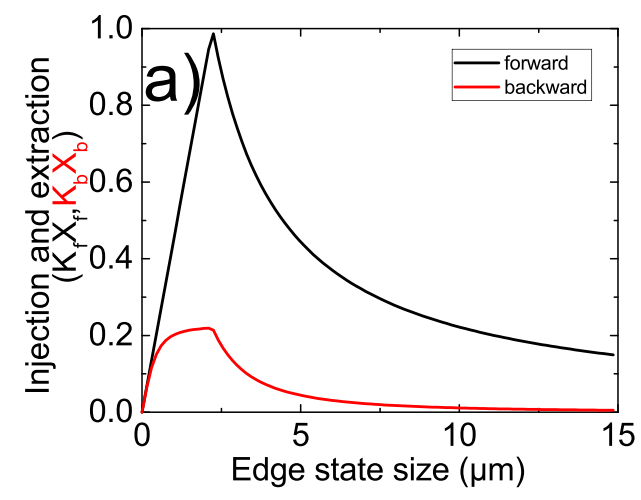

opposite direction, and the efficiency is given by $X_{f}=\min (1, w / l)$. In our case, it is difficult to reduce the edge state sufficiently, and we are dealing with the limit $l>w$. Thus, $K_{f} X_{f} \approx w / l$.

For the backward direction, the injection lead (the one marked output in Fig. 1) is perpendicular to the edge state that it injects, and the injection coefficient is therefore reduced because of the finite overlap integral

$$
K_{b} \approx \frac{\left|\int_{0}^{\infty} e^{i k y} e^{-y / l} d y\right|}{\int_{0}^{\infty} e^{-y / l} d y} \approx \frac{1}{\sqrt{1+k^{2} l^{2}}} \approx \frac{1}{k l}
$$

The extraction can be approximated as for the forward direction, and we obtain $K_{b} X_{b} \approx w / k l^{2}$. The propagation wavevector in the lead can vary, but we can assume it to be close to $w$ for estimation purposes. The dependence of both products $K_{f} X_{f}$ and $K_{b} X_{b}$ for forward and backward directions on the edge state size $l$ is plotted in Fig. 2(a). Both products decay for large $l$, but $K_{b} X_{b} \propto 1 / l^{2}$ decays faster than $K_{f} X_{f} \propto 1 / l$. The ratio (3) can be increased arbitrarily by increasing the path for the parasite signal $l_{o \rightarrow i}$. However, for the purposes of integration, the device size has to be kept minimal. This is why we assume a square-shaped device of the size $L$, which gives $l_{i \rightarrow o}=L$ and $l_{o \rightarrow i}=3 L$, and the first expression reduces to

$$
\frac{T_{F}}{T_{B}}=\frac{I_{i \rightarrow o}}{I_{o \rightarrow i}}=\frac{1}{k l} \exp (2 L / v \tau) .
$$

The reduction of the useful signal intensity is given by $I_{i \rightarrow o} / I_{i}=K_{f} X_{f} \exp \left(-l_{i \rightarrow o} / v \tau\right)$. The forward transmission of the useful signal can be up to $10 \%$. The use of polaritons allows us to selectively apply localized amplification to one of the channels by non-resonant pumping. ${ }^{77}$ This allows us to keep $I_{i \rightarrow o} / I_{i} \approx 1$, while reducing the other ratio by reducing $v$.

The efficiency of the device also depends on secondary parameters such as the orientation of the input and output waveguides. Figure 2(b) shows the injection efficiency as a function of injection angle $\theta_{i n}$. The black curve gives a simple efficiency estimate $K_{f} \propto \cos \theta_{\text {in }}$ corresponding to the projection of the injection region. The blue curve takes into account the diffraction, calculated similar to the single-slit diffraction case, which gives a coefficient $\left(l \sin \left(\pi w \sin \theta_{i n} / l\right) / \pi \sin \theta_{\text {in }}\right)^{2}$. For the output lead, numerical simulations have shown an optimal value of $\theta_{\text {out }} \approx 30^{\circ}$, providing alignment with the orientation of the link between the pillars at the zigzag edge, which improves the outcoupling.
FIG. 2. (a) The forward (black) and backward (red) transmission efficiency as a function of the edge state size $l$ for a fixed waveguide width $w$. (b) The injection efficiency as a function of the injection angle $\theta_{i n}$ : simple estimation (black), diffraction pattern (blue), and numerical simulations (red dots).

The localization length $l$ imposes a minimal bound on $L$ : $L>2 l$; otherwise, the edge state actually covers the whole sample and the chirality is suppressed. Our analysis leads to the following optimal device configuration: a square sample of polariton graphene with a size of the order of $3 l(10 \times 9$ unit cells) with the input waveguide parallel to one of the sides of the square, and the output waveguide oriented at $30^{\circ}$. The width of the leads $w \approx 2.2 \mu \mathrm{m}$ is roughly comparable to the pillar diameter.

Figures 3(a)-3(c) show an example of the simulation of the propagation of light through the optimized device. The simulations were based on the full Schrodinger equation (without the tight-binding approximation) with the parameters of GaAs-based patterned cavities. ${ }^{39}$ The equation reads

$$
\begin{aligned}
i \hbar \frac{\partial \psi_{ \pm}}{\partial t}= & -\frac{\hbar^{2}}{2 m} \Delta \psi_{ \pm}-\frac{i \hbar}{2 \tau} \psi_{ \pm}+\Delta_{z} \psi_{ \pm} \\
& +\beta\left(\frac{\partial}{\partial x} \mp i \frac{\partial}{\partial y}\right)^{2} \psi_{\mp}+U \psi_{ \pm}+\hat{P}
\end{aligned}
$$

where $\psi_{+}(\mathbf{r}, t), \psi_{-}(\mathbf{r}, t)$ are the two circular components, $m=5 \times 10^{-5} m_{e l}$ is the polariton mass, $\tau=30 \mathrm{ps}$ is the
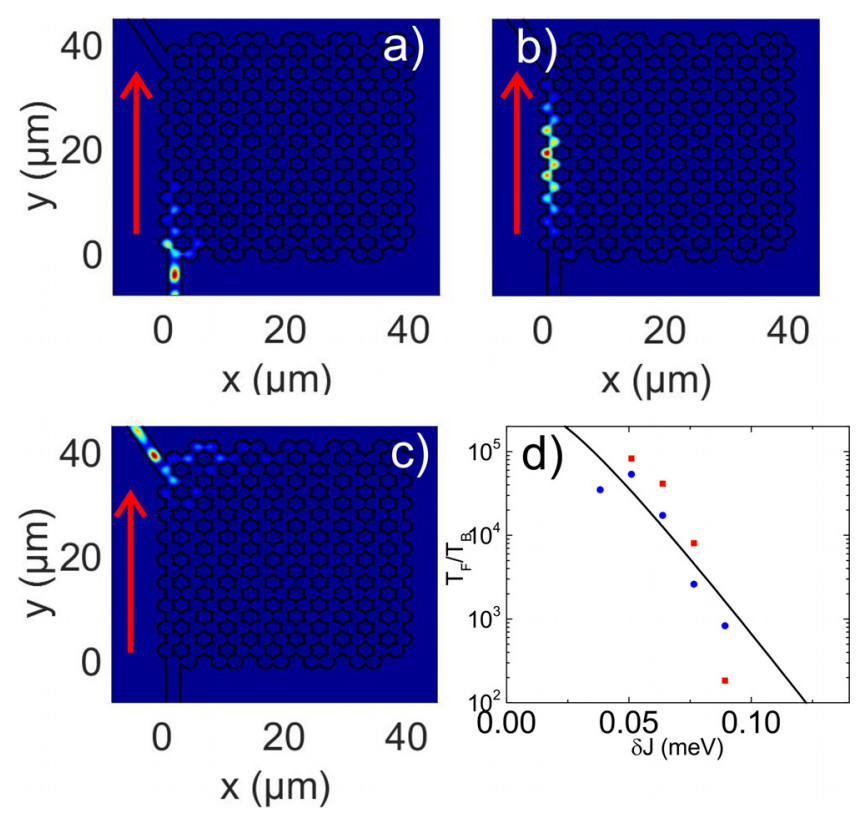

FIG. 3. (a)-(c) The functioning of the topological optical isolator device in a patterned microcavity (propagation in the forward direction): (a) injection $(t=0)$, (b) propagation ( $t=30 \mathrm{ps}$ ), and (c) extraction ( $t=60 \mathrm{ps}$ ). (d) Ratio of the forward to backward transmission coefficients as a function of the TE-TM splitting $\delta J$ : analytical estimation (black curve) and numerical simulations (non-amplified—blue; amplified—red). 
lifetime, and $\beta$ is the TE-TM coupling constant (corresponding to a $5 \%$ difference in the longitudinal and transverse masses). $\Delta_{z}$ is the Zeeman splitting, $U$ is the lattice potential, and $\hat{P}$ is the pump operator $(\tilde{P} \tau \propto 1)$. In some calculations, a localized (Gaussian RMS width $\sigma=1 \mu \mathrm{m}$ ) excitonic reservoir [marked with an orange spot in Fig. 1(a)], bringing a positive imaginary contribution $5 \times 10^{10} \mathrm{~s}^{-1}$ into $U(\mathbf{r})$, provides amplification ${ }^{77}$ (see below). We stress that circularpolarized non-resonant optical pumping can be used ${ }^{33}$ instead of an external magnetic field (which has to reach $9 \mathrm{~T}$ for the Zeeman splitting values used). The calculations on a $2^{9} \times 2^{9}$ grid were performed on the Graphics Processing Unit using nVidia CUDA.

Using these equations, we have first verified our analytical predictions for the group velocity and the gap size [red dots in Figs. 1(b) and 1(c)]. We see that these predictions are in good agreement with simulations. We have then carried out more preliminary simulations to determine the optimal structure parameters, for example, varying the injection angle. The results are shown in Fig. 2(b) with red dots. Again, a good agreement with simple analytical estimates is found.

In Figs. 3(a)-3(c), the contours of the pillars and the waveguides are shown with black lines and the colormap represents the total intensity of light as a function of spatial coordinates $\left|\psi_{+}(\mathbf{r})\right|^{2}+\left|\psi_{-}(\mathbf{r})\right|^{2}$. We see that the signal propagates from the input (panel a) into the output lead (panel c), staying within the spatial extension of the edge state $l$ (panel b). Finally, Fig. 3(d) shows the forward to backward transmission ratio which is the main figure of merit of the efficiency of the optical isolator as a device, plotted as a function of the TE-TM strength, calculated both analytically (black line) with Eq. (5) [an approximation for Eq. (3)], taking into account the injection and extraction efficiencies, the group velocity, and the finite lifetime, and numerically, without amplification (blue dots) and with amplification (red dots). The ratio $T_{F} / T_{B}$ first increases due to the decrease in the group velocity and then starts to decrease because of the increase in the efficiency of backward injection $K_{b}$. While the amplification allows us to compensate a part of the losses and obtain the forward transmission coefficient $I_{i \rightarrow o} / I_{i} \approx 1$, it cannot be used to increase much the isolation ratio; otherwise (for a 3-fold amplification increase), the device switches to the lasing regime, operating as a topological laser. ${ }^{10}$ The maximal achieved isolation ratio is $T_{F} / T_{B}=I_{i \rightarrow o} / I_{o \rightarrow i} \approx 8.2 \times 10^{4}$ or $49 \mathrm{~dB}$.

The choice of the GaAs-based realization implies low operating temperature [up to $50 \mathrm{~K}$ (Ref. 78)] and an operating wavelength of $\lambda \approx 783 \mathrm{~nm}$. The maximal bandwidth of the device is determined by the topological gap $E_{g} \approx 0.2 \mathrm{meV}$, giving $0.1 \mathrm{~nm}$. While being fairly small for purely optical purposes, this bandgap allows a modulation frequency of $300 \mathrm{GHz}$, which allows us to have optical computation schemes 30 times faster than the best current electronic schemes. The need to work at cryogenic temperature could be overcome by using structures based on widebandgap semiconductors ${ }^{79}$ or organic materials,${ }^{80}$ possibly allowing the implementation of room temperature polaritonics with a larger bandwidth.
To conclude, we have studied a practical application of polariton graphene: a topological optical isolator. The analytical estimations and numerical simulations show that the device should exhibit remarkable properties in terms of the isolation ratio $(49 \mathrm{~dB})$ and size $(40 \mu \mathrm{m})$ and a transmission close to unity.

We acknowledge the support of the project "Quantum Fluids of Light" (ANR-16-CE30-0021), of the ANR Labex Ganex (ANR-11-LABX-0014), and of the ANR Labex IMobS3 (ANR-10-LABX-16-01). D.D.S. acknowledges the support of IUF (Institut Universitaire de France).

${ }^{1}$ M. Z. Hasan and C. L. Kane, Rev. Mod. Phys. 82, 3045 (2010).

${ }^{2}$ K. v. Klitzing, G. Dorda, and M. Pepper, Phys. Rev. Lett. 45, 494 (1980).

${ }^{3}$ D. J. Thouless, M. Kohmoto, M. P. Nightingale, and M. den Nijs, Phys. Rev. Lett. 49, 405 (1982).

${ }^{4}$ C. L. Kane and E. J. Mele, Phys. Rev. Lett. 95, 146802 (2005).

${ }^{5}$ Z. Wang, Y. Chong, J. D. Joannopoulos, and M. Soljacic, Nature 461, 772 (2009).

${ }^{6}$ L. Lu, J. D. Joannopoulos, and M. Soljacic, Nat. Photonics 8, 821 (2014).

${ }^{7}$ D. Jalas, A. Petrov, M. Eich, W. Freude, S. Fan, Z. Yu, R. Baets, M. Popovic, A. Melloni, J. D. Joannopoulos, M. Vanwolleghem, C. R. Doerr, and H. Renner, Nat. Photonics 7, 579 (2013).

${ }^{8}$ Y. Wu, C. Li, X. Hu, Y. Ao, Y. Zhao, and Q. Gong, Adv. Opt. Mater. 5, 1700357 (2017).

${ }^{9}$ A. Politano, L. Viti, and M. S. Vitiello, APL Mater. 5, 035504 (2017).

${ }^{10}$ B. Bahari, A. Ndao, F. Vallini, A. El Amili, Y. Fainman, and B. Kanté, Science 358, 636 (2017).

${ }^{11}$ L. Pilozzi and C. Conti, Phys. Rev. B 93, 195317 (2016).

${ }^{12}$ J. Koo, J. Lee, and J. H. Lee, J. Lightwave Technol. 35, 2175 (2017).

${ }^{13}$ C. Ouyang, Z. Xiong, F. Zhao, B. Dong, X. Hu, X. Liu, and J. Zi, Phys. Rev. A 84, 015801 (2011).

${ }^{14}$ L. Lu and Z. Wang, e-print arXiv:1611.01998v1.

${ }^{15}$ M. Hafezi, E. A. Demler, M. D. Lukin, and J. M. Taylor, Nat. Phys. 7, 907 (2011).

${ }^{16}$ K. Lai, T. Ma, X. Bo, S. Anlage, and G. Shvets, Sci. Rep. 6, 28453 (2016).

${ }^{17}$ W. Qiu, Z. Wang, and M. Soljacic, Opt. Express 19, 22248 (2011).

${ }^{18}$ V. Peano, M. Houde, F. Marquardt, and A. A. Clerk, Phys. Rev. X 6, 041026 (2016).

${ }^{19}$ Z. Chai, X. Hu, F. Wang, X. Niu, J. Xie, and Q. Gong, Adv. Opt. Mater. 5, 1600665 (2017).

${ }^{20}$ J. Clark and G. Lanzani, Nat. Photonics 4, 438 (2010).

${ }^{21}$ X. Hu, P. Jiang, C. Ding, H. Yang, and Q. Gong, Nat. Photonics 2, 185 (2008).

${ }^{22}$ D. Leykam and Y. D. Chong, Phys. Rev. Lett. 117, 143901 (2016).

${ }^{23}$ X. Cheng, C. Jouvaud, X. Ni, S. H. Mousavi, A. Z. Genack, and A. B. Khanikaev, Nat. Mater. 15, 542 (2016).

${ }^{24}$ C. He, X. L. Chen, M. H. Lu, X. F. Li, W. W. Wan, X. S. Qian, R. C. Yin, and Y. F. Chen, Appl. Phys. Lett. 96, 111111 (2010).

${ }^{25}$ Z. Yu, G. Veronis, Z. Wang, and S. Fan, Phys. Rev. Lett. 100, 023902 (2008).

${ }^{26}$ M. F. Yanik, S. Fan, M. Soljačić, and J. D. Joannopoulos, Opt. Lett. 28, 2506 (2003).

${ }^{27}$ R. El-Ganainy and M. Levy, Opt. Lett. 40, 5275 (2015).

${ }^{28}$ H. Yokoi, Y. Shoji, and T. Mizumoto, Jpn. J. Appl. Phys., Part 1 43, 5871 (2004).

${ }^{29}$ T. Ozawa, H. M. Price, N. Goldman, O. Zilberberg, and I. Carusotto, Phys. Rev. A 93, 043827 (2016).

${ }^{30}$ D. Solnyshkov and G. Malpuech, C. R. Phys. 17, 920 (2016).

${ }^{31}$ G. Siroki, P. A. Huidobro, and V. Giannini, Phys. Rev. B 96, 041408 (2017).

${ }^{32}$ X. Zhou, Y. Wang, D. Leykam, and Y. D. Chong, New J. Phys. 19, 095002 (2017).

${ }^{33}$ O. Bleu, D. D. Solnyshkov, and G. Malpuech, Phys. Rev. B 95, 115415 (2017).

${ }^{34}$ N. Chamanara, S. Taravati, Z.-L. Deck-Léger, and C. Caloz, Phys. Rev. B 96, 155409 (2017).

${ }^{35}$ A. V. Nalitov, D. D. Solnyshkov, and G. Malpuech, Phys. Rev. Lett. 114, 116401 (2015).

${ }^{36}$ D. A. Kuzmin, I. V. Bychkov, V. G. Shavrov, and V. V. Temnov, ACS Photonics 4, 1633 (2017). 
${ }^{37}$ M. C. Rechtsman, J. M. Zeuner, Y. Plotnik, Y. Lumer, D. Podolsky, F. Dreisow, S. Nolte, M. Segev, and A. Szameit, Nature 496, 196 (2013).

${ }^{38}$ Z.-Y. Li, R.-J. Liu, L. Gan, J.-X. Fu, and J. Lian, Int. J. Mod. Phys. B 28, 1441010 (2014).

${ }^{39}$ T. Jacqmin, I. Carusotto, I. Sagnes, M. Abbarchi, D. D. Solnyshkov, G. Malpuech, E. Galopin, A. Lemaître, J. Bloch, and A. Amo, Phys. Rev. Lett. 112, 116402 (2014).

${ }^{40}$ L. B. Ma, S. L. Li, V. M. Fomin, M. Hentschel, Y. Gotte, J. B. A. N. Yin, M. R. Jorgensen, and O. G. Schmidt, Nat. Commun. 7, 10983 (2016).

${ }^{41}$ F. Auracher and H. Witte, Opt. Commun. 13, 435 (1975).

${ }^{42}$ S. K. Ibrahim, S. Bhandare, D. Sandel, H. Zhang, and R. Noe, Electron. Lett. 40, 1293 (2004).

${ }^{43}$ Z. Yu and S. Fan, IEEE J. Sel. Top. Quantum Electron. 16, 459 (2010).

${ }^{44}$ D. L. Sounas and A. Alù, ACS Photonics 1, 198 (2014).

${ }^{45}$ T. Shintaku, Appl. Phys. Lett. 66, 2789 (1995).

${ }^{46}$ H. Shimizu, Proc. SPIE 8813, 88132C (2013).

${ }^{47}$ J. Ma, X. Xi, Z. Yu, and X. Sun, Appl. Phys. Lett. 108, 151103 (2016).

${ }^{48}$ K. Wang, Y. Wang, S. Gao, A. Nirmalathas, C. Lim, K. Alameh, H. Li, and E. Skafidas, IEEE Photonics Technol. Lett. 29, 1261 (2017).

${ }^{49}$ D. Huang, P. Pintus, C. Zhang, Y. Shoji, T. Mizumoto, and J. E. Bowers, IEEE J. Sel. Top. Quantum Electron. 22, 271 (2016).

${ }^{50}$ N. Bahlmann, M. Lohmeyer, M. Wallenhorst, H. Dötsch, and P. Hertel, Opt. Quantum Electron. 30, 323 (1998).

${ }^{51}$ Y. Sun, H. Zhou, X. Jiang, Y. Hao, J. Yang, and M. Wang, J. Opt. 12, 015403 (2010).

${ }^{52}$ Y. Shoji and T. Mizumoto, Opt. Express 15, 639 (2007).

${ }^{53}$ A. Kavokin, J. J. Baumberg, G. Malpuech, and F. P. Laussy, Microcavities (Oxford University Press, 2011).

${ }^{54}$ N. Masumoto, N. Y. Kim, T. Byrnes, K. Kusudo, A. Loeffler, S. Hoefling, A. Forchel, and Y. Yamamoto, New J. Phys. 14, 065002 (2012).

${ }^{55}$ M. Galbiati, L. Ferrier, D. D. Solnyshkov, D. Tanese, E. Wertz, A. Amo, M. Abbarchi, P. Senellart, I. Sagnes, A. Lemaitre, E. Galopin, G. Malpuech, and J. Bloch, Phys. Rev. Lett. 108, 126403 (2012).

${ }^{56}$ E. A. Cerda-Méndez, D. Sarkar, D. N. Krizhanovskii, S. S. Gavrilov, K. Biermann, M. S. Skolnick, and P. V. Santos, Phys. Rev. Lett. 111, 146401 (2013).

${ }^{57}$ E. A. Cerda-Mendez, D. N. Krizhanovskii, K. Biermann, R. Hey, M. S. Skolnick, and P. V. Santos, New J. Phys. 14, 075011 (2012).

${ }^{58}$ D. N. Krizhanovskii, E. A. Cerda-Méndez, S. Gavrilov, D. Sarkar, K. Guda, R. Bradley, P. V. Santos, R. Hey, K. Biermann, M. Sich, F. Fras, and M. S. Skolnick, Phys. Rev. B 87, 155423 (2013).

${ }^{59}$ D. Sanvitto, S. Pigeon, A. Amo, D. Ballarini, M. De Giorgi, I. Carusotto, R. Hivet, F. Pisanello, V. G. Sala, P. S. S. Guimaraes, R. Houdre, E. Giacobino, C. Ciuti, A. Bramati, and G. Gigli, Nat. Photonics 5, 610 (2011).
${ }^{60}$ A. Askitopoulos, H. Ohadi, A. V. Kavokin, Z. Hatzopoulos, P. G. Savvidis, and P. G. Lagoudakis, Phys. Rev. B 88, 041308 (2013).

${ }^{61}$ T. Karzig, C.-E. Bardyn, N. H. Lindner, and G. Refael, Phys. Rev. X 5, 031001 (2015).

${ }^{62}$ C.-E. Bardyn, T. Karzig, G. Refael, and T. C. H. Liew, Phys. Rev. B 91, 161413 (2015).

${ }^{63}$ C.-E. Bardyn, T. Karzig, G. Refael, and T. C. H. Liew, Phys. Rev. B 93, 020502 (2016).

${ }^{64}$ Y. V. Kartashov and D. V. Skryabin, Optica 3, 1228 (2016).

${ }^{65}$ V. M. Kovalev, I. G. Savenko, and I. V. Iorsh, J. Phys.: Condens. Matter 28, 105301 (2016).

${ }^{66}$ D. R. Gulevich, D. V. Skryabin, A. P. Alodjants, and I. A. Shelykh, Phys. Rev. B 94, 115407 (2016).

${ }^{67}$ D. R. Gulevich, D. Yudin, D. V. Skryabin, I. V. Iorsh, and I. A. Shelykh, Sci. Rep. 7, 1780 (2017).

${ }^{68}$ H. Sigurdsson, G. Li, and T. C. H. Liew, Phys. Rev. B 96, 115453 (2017).

${ }^{69}$ A. V. Nalitov, T. C. H. Liew, A. V. Kavokin, B. L. Altshuler, and Y. G. Rubo, Phys. Rev. Lett. 119, 067406 (2017).

${ }^{70}$ M. Milicevic, T. Ozawa, P. Andreakou, I. Carusotto, T. Jacqmin, E. Galopin, A. Lemaitre, L. Le Gratiet, I. Sagnes, and J. Bloch, 2D Mater. 2, 034012 (2015).

${ }^{71}$ P. St-Jean, V. Goblot, E. Galopin, A. Lemaitre, T. Ozawa, L. Le Gratiet, I. Sagnes, J. Bloch, and A. Amo, Nat. Photonics 11, 651 (2017).

${ }^{72}$ M. Milićević, T. Ozawa, G. Montambaux, I. Carusotto, E. Galopin, A. Lemaitre, L. Le Gratiet, I. Sagnes, J. Bloch, and A. Amo, Phys. Rev. Lett. 118, 107403 (2017).

${ }^{73}$ S. Klembt, T. H. Harder, O. A. Egorov, K. Winkler, H. Suchomel, J. Beierlein, M. Emmerling, C. Schneider, and S. Höfling, Appl. Phys. Lett. 111, 231102 (2017).

${ }^{74}$ F. D. M. Haldane and S. Raghu, Phys. Rev. Lett. 100, 013904 (2008).

${ }^{75}$ A. V. Nalitov, G. Malpuech, H. Terças, and D. D. Solnyshkov, Phys. Rev. Lett. 114, 026803 (2015).

${ }^{76}$ D. Solnyshkov, A. Nalitov, B. Teklu, L. Franck, and G. Malpuech, Phys. Rev. B 93, 085404 (2016).

${ }^{77}$ E. Wertz, A. Amo, D. D. Solnyshkov, L. Ferrier, T. C. H. Liew, D. Sanvitto, P. Senellart, I. Sagnes, A. Lemaitre, A. V. Kavokin, G. Malpuech, and J. Bloch, Phys. Rev. Lett. 109, 216404 (2012).

${ }^{78}$ E. Wertz, L. Ferrier, D. D. Solnyshkov, P. Senellart, D. Bajoni, A. Miard, A. Lemaître, G. Malpuech, and J. Bloch, Appl. Phys. Lett. 95, 051108 (2009).

${ }^{79}$ S. Christopoulos, G. B. H. von Högersthal, A. J. D. Grundy, P. G. Lagoudakis, A. V. Kavokin, J. J. Baumberg, G. Christmann, R. Butté, E. Feltin, J.-F. Carlin, and N. Grandjean, Phys. Rev. Lett. 98, 126405 (2007).

${ }^{80}$ D. M. Coles, N. Somaschi, P. Michetti, C. Clark, P. G. Lagoudakis, P. G. Savvidis, and D. G. Lidzey, Nat. Mater. 13, 712 (2014). 